University of South Carolina

Scholar Commons

2000

\title{
Modeling the Effect of Plasticizer on the Viscoelastic Response of Crosslinked Polymers Using the Tube-Junction Model
}

\author{
P. P. Simon \\ Harry J. Ploehn \\ University of South Carolina - Columbia, ploehn@cec.sc.edu
}

Follow this and additional works at: https://scholarcommons.sc.edu/eche_facpub

Part of the Mechanical Engineering Commons, and the Mechanics of Materials Commons

\footnotetext{
Publication Info

Published in Journal of Rheology, Volume 44, Issue 2, 2000, pages 169-183.

(C) Journal of Rheology 2000, American Institute of Physics

Simon, P. P. \& Ploehn, H. J. (2000). Modeling the effect of plasticizer on the viscoelastic response of crosslinked polymers using the tube-junction model. Journal of Rheology, 44(2), 169-183.

http://dx.doi.org/10.1122/1.551082

http://scitation.aip.org/content/sor/journal/jor2/44/2/10.1122/1.551082
}

This Article is brought to you by the Chemical Engineering, Department of at Scholar Commons. It has been accepted for inclusion in Faculty Publications by an authorized administrator of Scholar Commons. For more information, please contact digres@mailbox.sc.edu. 


\title{
Modeling the effect of plasticizer on the viscoelastic response of crosslinked polymers using the tube-junction model
}

\author{
Philip P. Simon and Harry J. Ploehn ${ }^{\text {a) }}$ \\ Department of Chemical Engineering, University of South Carolina, \\ Swearingen Engineering Center, Columbia, South Carolina 29208
}

(Received 27 October 1998; final revision received 8 December 1999)

\begin{abstract}
Synopsis
Plasticizers modify the mechanical properties of polymeric materials. The effects of plasticizers on glass transition temperatures can be most clearly observed in isochronal temperature sweep profiles of viscoelastic dynamic moduli. However, no simple mathematical models of plasticization are available to those who wish to design and employ plasticized materials in specific applications. We extend a phenomenological, molecular-level model (known as the tube-junction model) for crosslinked polymers to describe the effect of plasticizers on dynamic moduli. We show that the increase in free volume fraction due to the presence of the plasticizer can account for the shift in the glass transition in dynamic moduli. We also show that the secondary effects of plasticizers on the shape of the temperature sweep profiles can be explained in terms of increased width of the distribution of activation energies associated with intermolecular frictional forces. (C) 2000 The Society of Rheology. [S0148-6055(00)00702-1]
\end{abstract}

\section{INTRODUCTION}

Polymer materials used in today's applications are modified from their pure forms to enable them to perform specialized tasks. Addition of impact modifiers for crack resistance, fillers for increased stiffness, and plasticizers for increased flexibility are some examples of these modifications. Often, the environment modifies the polymers in the course of their service by promoting the loss or uptake of plasticizer. An important example is the uptake of water by epoxy resin composites used for structural components in watercraft or offshore installations. The viscoelastic modulus of an epoxy resin depends on the frequency of the mechanical loading, the ambient temperature, and the water content in the bulk of the resin. Epoxy resin absorbs water, which alters the time-temperature relationship of the modulus through plasticization. In order to predict the behavior of structures made from crosslinked polymers or to evaluate the properties of new polymer formulations, it is necessary to understand and characterize the effect of plasticizer on the viscoelastic response of crosslinked polymers.

Plasticizers produce a number of effects on polymers. The most prominent of these is the decrease in the glass transition temperature $T_{g}$. The change in $T_{g}$ is observed in many experiments such as dilatometry, three-point bending, and dynamic mechanical analysis. The fundamental processes responsible for the various manifestations of glass transitions are still not completely understood. A number of theories exist that explain

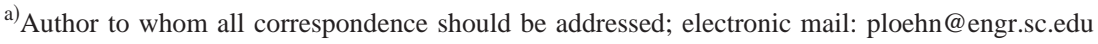


specific isolated features of the transition, as we will see shortly. However, none of them provide a rigorous analysis of the effect of plasticizers on the glass transition or of glass transitions in dynamic mechanical analysis. Kelley and Bueche (1961) successfully modeled the decrease in the dilatometric $T_{g}$ using the free volume concept of the well-known Williams-Landau-Ferry theory [Ferry (1980)]. Although their model was developed for plasticizers that have glass transition temperatures, others [e.g., Browning et al. (1977)] have applied it with reasonable success for plasticization with nonglass-transitioning diluents such as water.

Ferry (1980) enumerates a number of secondary effects of plasticizers on the dynamic moduli of polymers. One such effect is the change in the shape of the viscoelastic functions. Figure 17-44 of Ferry (1980) shows typical effects of plasticizer on the dynamic moduli reported in a constant frequency (isochronal) temperature sweep. The isochronal temperature sweep profile shows the change in the dynamic moduli at a constant frequency over a range of temperatures. The changes in the shape of the viscoelastic functions include decreased steepness of the storage modulus and decreased sharpness of the loss modulus peak in the glass transition zone. Although these effects of plasticizers on dynamic moduli are well known, no existing model can replicate these effects.

Spring-and-dashpot models have been used successfully to characterize the time and frequency response of the moduli of crosslinked polymers. These models, however, require data at constant temperature and plasticizer content. Furthermore, data used by these models generally cannot be measured over a sufficient range of frequency or time because of practical limitations. The models are most often fitted to master curves constructed using measurements at different temperatures. Such measurements must necessarily be at constant plasticizer content. Consequently, spring-and-dashpot models cannot model or predict the effect of temperature or plasticizer. Although there have been a number of molecular models for the viscoelasticity of polymers, none have explicitly accounted for the effect of temperature or plasticizers other than from a scaling approach using the shift factors [Ferry (1980)].

We have developed a phenomenological, molecular-level model [Simon and Ploehn (1997)] that describes the viscoelasticity of crosslinked polymers. We will refer to this model as the tube-junction (TJ) model because it describes the dynamics of a flexible chain constrained by entanglement tubes and junctions. To our knowledge, the TJ model is unique in its ability to describe large changes in the moduli with variations in temperature as well as with frequency. The model can qualitatively characterize the isochronal temperature sweep of a crosslinked polymer, revealing explicitly the dynamic glass transition temperature. In addition, the model qualitatively describes the isothermal frequency sweeps of the polymer. Therefore, the TJ model may serve as a useful tool to examine the effect of plasticizers on the change in the viscoelastic behavior of crosslinked polymers and their composites. The model is limited by the phenomenological descriptions of the various molecular-level forces, which introduce parameters that must be tuned using an isochronal temperature sweep profile. The model also assumes only a single relaxation process associated with axial "reptative" motion of the polymer. These features limit the quantitative accuracy of the model, especially in the glass transition regime. However, some modifications can be made to the model to improve its quantitative accuracy.

In this paper, we extend the TJ model to describe the effects of plasticizers on isochronal temperature sweeps and isothermal frequency sweeps for crosslinked polymers. We assume that plasticizers have the same effect on the polymer as temperature, namely, to change the free volume content and thus the friction coefficient associated with relative 
chain motion. The decrease in the magnitude of the monomeric friction coefficient with plasticization is well known [Ferry (1980)], leading to a decrease in the viscoelastic glass transition temperature. Following Kelley and Bueche (1961), we assume that the free volumes of polymer and plasticizer are additive. Based on this assumption, we use the extended TJ model to predict the effect of water uptake on temperature and frequency sweeps for epoxy resins.

\section{A. Glass transitions}

Before introducing our theory to account for plasticizer effects on the properties of crosslinked polymers, we will first comment on two different viewpoints of the glass transition and explain their relationships to this work.

There are a number of theories proposed to describe various aspects of the glass transition. These theories propose relaxational effects [Roberts and White (1973); Williams et al. (1955); Kauzmann (1948)] as well as thermodynamic phase transitions [Gibbs and DiMarzio (1958); Adam and Gibbs (1965); Cohen and Grest $(1979,1981,1984)]$ as the fundamental mechanism controlling the glass transition. The nature of the thermodynamic phase transition, whether it is a first or second order transition, is also an unsettled issue. However, neither the relaxational nor the thermodynamic approaches can independently account for all of the different features of the transition. Attempts at postulating a purely relaxational mechanism encounter the Kauzmann paradox [Kauzmann (1948)] in which a supercooled glass appears to have a lower entropy than the crystalline form of the same material. Attempts at postulating only a thermodynamic phase transition at some temperature $T_{g, t}$ below the observed dilatational glass transition temperature $T_{g \text {,dil }}$ encounter difficulties in explaining the observed thermal history dependence. The nonequilibrium nature of the glassy state and the thermal history dependence of $T_{g}$ have motivated many nonequilibrium theories [Moynihan and Lesikar (1981); Astarita et al. (1989)]. Donth (p. 138, 1992) suggests that the kinetic and thermodynamic effects are probably independent and coexisting, while DiMarzio (1981) suggests that the kinetic and thermodynamic effects are probably intimately connected. No consensus in this area has been achieved.

We take the view that both thermodynamic and kinetic effects coexist in the material, although the kinetics of the molecular mechanisms obscures the measurement of the thermodynamic phase transition in dilatation. First, we assume that a thermodynamic phase transition (whether of the first order or second order) changes the thermal expansion coefficient of the material at some temperature $T_{g, t}$. We ignore the effect of pressure for now. Above $T_{g, t}$, the material has a rubbery thermal expansion coefficient, and below $T_{g, t}$ the material is in a glassy state with a glassy thermal expansion coefficient.

Unfortunately, the value of $T_{g, t}$ is not easily measured. The large time scales associated with the molecular motion at low temperatures close to the glassy state obscure the observation of $T_{g, t}$ in a typical dilatometric measurement. The glass transition temperature measured in a dilatometric experiment, $T_{g \text {, dil }}$, is consequently higher than $T_{g, t}$ because large frictional forces prevent the material from reaching equilibrium in the time scale of the experiment. Motivated primarily by lack of a better estimate of $T_{g, t}$, we use the dilatometric $T_{g \text {,dil }}$ as a measure of $T_{g, t}$. This choice also relies on the fact that, for all practical purposes, the material behaves as a glass below the dilatometric $T_{g \text {,dil }}$, just as it would below $T_{g, t}$, because molecular motions are very slow in that temperature regime. The error associated with this assumption will become significant only when extremely low frequencies or large time scales are considered. 


\section{THEORY}

The TJ model relates the dynamic moduli of a crosslinked polymer to its thermodynamic state, such as temperature, through the friction coefficient $\zeta$. The model assumes that $\zeta$ is a function of the free volume fraction in the material. Although free volume fraction is a thermodynamic property of the material [Simha and Somcynsky (1969); Simha (1977); Robertson (1992)], the absolute magnitudes of free volume fraction calculated from dynamic measurements do not correspond to realistic definitions of the free volume fraction [Roberts and White (1973); Ferry (1980)]. Conditions such as temperature, pressure, and diluent content change the thermodynamic state of the material and the corresponding free volume fraction. A diluent in the context of thermodynamics is equivalent to a plasticizer in the dynamics context. We will use the terms diluent and plasticizer interchangeably.

Ideally, one needs an equation of state that describes the variation of the free volume fraction as a function of temperature and plasticizer content over all possible states of the material. To date, there are no theories that provide this information. Nevertheless, there are simpler theories that can be used to account for the thermodynamic effect of plasticizers on the free volume. One such theory, the Kelley-Bueche model (1961), shows that changes in the dilatometric glass transition temperature can be modeled by accounting for the change in the free volume fraction resulting from the presence of plasticizer and its thermal expansion coefficient. We follow this course here. First, we review the essential elements of the TJ model [Simon and Ploehn (1997)]. Next, we describe modifications of the free volume expressions within the model that enable predictions of the effect of plasticizer on dynamic mechanical properties.

\section{A. Tube-junction model: Background}

In the TJ model, each strand of a crosslinked polymer chain is constrained inside an entanglement tube having a cross-sectional area that varies along the length of the tube. Neighboring chains, treated as a continuum, define the surface of the tube. Thermal fluctuations permit the chain to act like an entropy spring that takes up all conformations within the confines of this tube. At entanglement junctions, the tube cross section becomes comparable to the dimensions of the chain cross section, so chain motions are restricted to energy-dissipating axial translation. The junctions divide the entanglement tube into subtubes and the chain into smaller entropy springs that are "daisy chained" to one another. The end-to-end vectors of the subtubes have different orientations within the continuum.

Three types of forces act upon the chain inside the subtube. One is a cohesive force arising due to attractive intermolecular interactions between the chain and the tube walls. Upon perturbation of the equilibrium state, this force tends to reduce the intermolecular distances by either contracting the tube dimensions or by drawing additional chain length into the tube. Both processes occur simultaneously at appropriate rates. The former process results in the glassy relaxations, such as the beta relaxation, representing an activated process associated with chemical bond rotations of the molecules in the surrounding medium. The latter results in the alpha relaxation, which is observed in dynamic mechanical analysis as the dynamic glass transition. Thus, in the initial stages of the deformation, the cohesive force is the driving force for the two relaxations. Another force, the frictional force between the reptating chain and the tube at the entanglement junction, dissipates the translational energy. The final force is the entropic force that acts between the end points of a chain in a subtube. The end points that delimit the subtube can be either entanglement junctions or crosslink points. 
An externally applied deformation on the boundaries of the material produces an affine deformation of the tubes as well as of the coordinates of the tube ends. The deformations of the tubes vary with their orientation in the strain field. Simon and Ploehn (1997) simplified the problem by treating uniaxial elongational deformation of a two subtube system configured with one subtube oriented axially and the other transversely to the axis of deformation. The cohesive force is modeled in this special case as proportional to the dilatation created in the material. The chain is drawn into the tube to reduce the extra dilatational space created due to deformation. The end-to-end distance of this chain segment changes with the deformation of the end points of its subtube. If the chain was originally at equilibrium, the elongation of the subtube will also create an entropic force to increase the chain length in the subtube to a new equilibrium length. The resulting motion of the chain reduces both the cohesive and entropic forces with time. The material reaches equilibrium when the net force on the chain reduces to zero.

The approximation to a two-tube system (one axial and one transverse) reduces the model to a single relaxation mechanism for chain translation. A more realistic model would consider a distribution of tube orientations in the strain field. In addition to the distribution in tube orientations, one can also have a distribution in the frictional coefficient due to density or free volume fluctuations in the material. We will apply this latter modification in this analysis.

A momentum balance on a chain segment inside an entanglement junction

$$
F^{c}(t)+F_{a}^{s}(t)-F_{t}^{s}(t)+F^{v}(t)=0
$$

provides the variation of the longitudinal (uniaxial) force per unit area as a function of time and the corresponding time-dependent uniaxial elongation modulus

$$
E(t)=\frac{\bar{n}\left[F^{c}(t)+F_{a}^{s}(t)\right]}{\varepsilon} .
$$

Here superscripts $c, s$, and $v$ on the forces $F$ stand for cohesive, entropic, and viscous, respectively. Subscripts $a$ and $t$ stand for axial and transverse, respectively. Various phenomenological and approximate models [described in detail by Simon and Ploehn (1997)] provide the cohesive force

$$
F^{c}(t)=\frac{3 K}{\bar{n}}\left\{\varepsilon[1-2 \nu(t)]-\frac{x(t)}{l_{0}}\right\},
$$

the entropic force of rubber elasticity

$$
F_{l}^{s}(t)=-\frac{k T}{l_{0}}\left\{\frac{1}{2}\left(\alpha^{2}-\frac{1}{\alpha}\right)-\frac{x(t)}{l_{0}}\left(\alpha^{2}-\frac{1}{4 \alpha}\right)\right\},
$$

and the dissipative frictional force

$$
F_{l}^{v}(t)=-\zeta u(t)=-\zeta \frac{d x(t)}{d t}
$$

The frictional force depends on the frictional coefficient

$$
\zeta=\zeta_{0} \exp \left(\frac{b}{f_{v}}\right)
$$


which varies with the local free volume fraction $f_{v}$. In these equations, $\varepsilon$ is the applied uniaxial strain, $x(t)$ is the displacement of the chain in the axial tube, $l_{0}$ is the equilibrium length of the chain in a subtube, $\alpha=1+\varepsilon, K$ is the bulk modulus of the material, $\bar{n}$ is the number of tubes or junctions per unit cross-sectional area, and $\zeta_{0}$ is a preexponential friction coefficient. The time-varying Poisson's ratio in the glassy regime $\nu(t)$ given by

$$
\nu(t)=\nu_{\infty}-\left(\nu_{\infty}-\nu_{0}\right) \int_{0}^{\infty} \exp \left[-B_{c}(T) t\right] P\left(H_{c}\right) d H_{c}
$$

characterizes the glassy relaxations, such as the beta relaxation. Here $\nu_{\infty}$ is the ultimate glassy Poisson's ratio reached after all possible bond rotations are complete, and $\nu_{0}$ is the initial glassy Poisson's ratio before any relaxations have occurred. This latter value is set to zero. $P\left(H_{c}\right)$ is a probability distribution of the activation energies for transitions that occur between initial glassy Poisson's ratio $\nu_{0}$ and the ultimate glassy Poisson's ratio $\nu_{\infty}$. The model assumes that the relaxations occur as activated processes with rate constants given by

$$
B_{c}(t)=B_{c 0} \exp \left(-\frac{H_{c}}{R T}\right)
$$

where $B_{c 0}$ is a preexponential rate constant.

If the material has a distribution in the free volume, there will be a distribution in the response of chains within the material. A volume averaged elongational modulus that accounts for a distribution in the free volume may be written as

$$
\bar{E}(t)=\int_{0}^{\infty} E(t) P\left(f_{v}\right) d f_{v},
$$

where $P\left(f_{v}\right)$ is the probability distribution of the free volume fraction in the material at any given state of temperature and plasticizer. The model uses eight parameters and two material property functions $P\left(H_{c}\right)$ and $P\left(f_{v}\right)$. Introduction of the material property distribution functions introduces multiple relaxation times into the model.

$P\left(H_{c}\right)$ depicts the activation energy distribution function in the bond relaxations characteristic of the glassy state. As explained elsewhere [Simon and Ploehn (1997)], the population distribution of the activation energy of transitions should be determined independently, perhaps through infrared spectroscopy. In the absence of this information, we describe the distribution by

$$
P\left(H_{c}\right)=\frac{\exp \left(-\frac{H_{c}}{H^{*}}\right)+\exp \left[\frac{-\left(H_{c}-\bar{H}_{c}\right)^{2}}{2 \sigma_{H c}^{2}}\right]}{H^{*}+\sqrt{2 \pi} \sigma_{H c}}
$$

as a combination of a normal distribution of standard deviation $\sigma_{H c}$ about a mean value $\bar{H}_{c}$ and an exponential decay distribution with a decay constant $H^{*}$.

$P\left(f_{v}\right)$ depicts the variation of the free volume fraction as a function of temperature around a mean value. The probability distribution of the fractional free volume in the Simha-Somcynski model (1969) can be represented by the relatively simple gamma distribution [Robertson (1992)], 


$$
P\left(f_{v}\right)=\frac{\chi}{\Gamma(u)}\left(\chi f_{v}\right)^{u-1} e^{-\chi f_{v}}
$$

Here $\Gamma(u)$ is the gamma function, and $u$ and $\chi$ are parameters whose values are determined from

$$
\frac{u}{\chi}=\bar{f}_{v}, \quad \frac{u}{\chi^{2}}=\sigma_{f v}^{2},
$$

where $\bar{f}_{v}$ and $\sigma_{f v}^{2}$ are the mean and mean-squared fluctuations in the free volume. We use this function to describe the free volume distribution in an inhomogeneous material.

Due to lack of a rigorous thermodynamic equation of state over the whole temperature range, we use the empirical model

$$
\bar{f}_{v}=f_{v 0}=\alpha_{g} T+\left(\alpha_{r}-\alpha_{g}\right) \times\left(T-T_{g \mathrm{dil}}\right) \times \operatorname{Heaviside}\left(T-T_{g \mathrm{dil}}\right)
$$

to describe the variation of the mean free volume fraction $\bar{f}_{v}$ as a function of temperature. The subscripted $\alpha$ are thermal expansion coefficients with subscripts $g$ and $r$ referring to glassy and rubbery states, respectively. The absolute value of the free volume fraction at any given temperature is unknown and therefore $f_{v 0}$, the free volume fraction at $0 \mathrm{~K}$, is arbitrarily chosen to be zero. The transition from glassy to rubbery thermal expansion is assumed to occur at the dilatational glass transition temperature $T_{g}$, dil which approximates the thermodynamic glass transition as discussed earlier. The Heaviside $(\bullet)$ function has a value of 1 when the argument is positive and zero for all other values. The local free volume fraction at any point is obtained as a probability from Eqs. (11)-(13).

Four out of the eight parameters of the model have to be fitted using an isochronal temperature sweep. The isochronal data are ideal for the fitting because they include information about all the characteristic relaxations but unmodified by time-temperature rescaling. Parameter selection is discussed in detail elsewhere [Simon and Ploehn (1997)]. Using the parameter set from the isochronal fit, the model can be used to predict the frequency sweeps of the polymer at any given temperature.

\section{B. Tube-junction model: Effect of plasticizers}

Next, we describe our modification of the free volume expression in the TJ model to account for the effect of plasticizer. Following Kelley and Bueche (1961), we assume that the free volume fractions of the polymer and diluent constituents are additive in proportion to their bulk volume fractions. We retain our earlier definitions of the free volume fraction for the polymer [Eq. (13)], using the adjustable parameters $\zeta_{0}$ and $b$ in Eq. (6) to correct for the errors in the assumed magnitudes of $f_{v 0}$.

The expression for the free volume fraction are then given by

$$
f_{v}=\phi_{p} f_{v \text { polymer }}+\left(1-\phi_{p}\right) f_{v \text { diluent }}
$$

where $\phi_{p}$ is the bulk volume fraction of the polymer. Here $f_{v \text { polymer }}$ is given by Eq. (13), and $f_{v \text { diluent }}$ is given by

$$
f_{v \text { diluent }}=\alpha_{d} \times\left(T-T_{\text {md }}\right) \times \operatorname{Heaviside}\left(T-T_{\text {md }}\right)
$$


where $\alpha_{d}$ is the thermal expansion coefficient of the diluent in the polymer and $T_{\mathrm{md}}$ is the glass transition temperature of the diluent. Hypothetical $T_{g} \mathrm{~s}$ are used if the diluent crystallizes before supercooling [Ferry (1980)].

In the case of a material with nanoscale inhomogeneity, Eq. (14) gives the mean value $\bar{f}_{v}$ of the distribution in the free volume fraction. The probability of finding a given value of the free volume fraction $f_{v}$ at a given location is then described by Eqs. (11) and (12). The response of the polymer chains at any location is based on the local value of the free volume fraction. The net response of the material is obtained by integrating the response over the whole spectrum of free volume fractions [Eq. (9)].

\section{RESULTS AND DISCUSSION}

\section{A. System}

We compare predictions of the TJ model with experimental data [Wang and Ploehn (1996)] for the dynamic moduli of diglycidyl ether of bisphenol epoxy cured with triethylene tetramine. Henceforth, we will refer to this resin as the WP (Wang-Ploehn) resin. Their measurements included temperature sweep isochrones for dry and water-immersed samples of the resin. All of the experimental isochrones considered here were measured at a frequency of $10 \mathrm{rad} / \mathrm{s}$.

\section{B. Fitting the TJ model to dry resin data}

Table I lists the TJ model parameters chosen to characterize the dry resin isochronal temperature sweep data. All the parameters in Tables Ia-c, except the decay constant $H^{*}$, are chosen from data other than the isochronal temperature sweep. Most of the molecular-level parameters are the same as those used by Simon and Ploehn (1997) for another epoxy resin [Gupta et al. (1985)]. We use the value of the activation energy obtained form a shift factor analysis of a master curve $(70.7 \mathrm{~kJ} / \mathrm{mol})$ as the mean activation energy $\bar{H}_{c}$ of the $\beta$ transition. This value is close to the value used previously (63 $\mathrm{kJ} / \mathrm{mol}$ ) for the same resin cured with a different curing agent [Gupta et al. (1985)]. For the sake of consistency in the shape of the activation energy distribution profile, we scale the parameters $\sigma_{H c}$ and $H^{*}$ relative to the new value of $\bar{H}_{c}$. In addition, we use a value of dilatational $T_{\mathrm{dg}}=387 \mathrm{~K}$ [Kaplan (1991)].

The four parameters $n_{m}, b, \zeta_{0}$, and $B_{\text {co }}$ are adjusted by matching the temperature sweep profile of the data with the model at specific points. The magnitude of the $E^{\prime}$ in the rubbery zone sets the value of $n_{m}$, the slope of $E^{\prime}$, and the $\alpha$ peak temperature of $E^{\prime \prime}$ in the transition zone set the value for $b$ and $\zeta_{0}$, and the $\beta$ peak temperature of the $E^{\prime \prime}$ in the glassy zone sets the value for $B_{\mathrm{co}}$. The selection of these parameters for the WP resin follows the fitting procedure discussed in detail elsewhere [Simon and Ploehn (1997)]. The choice in the magnitudes of $b$ and $\zeta_{o}$, parameters in the friction coefficient expression are not without ambiguity.

Figure 1 shows the fit of the model to the dry resin data. The isochrone shows the glassy relaxations with the $\beta$ transition at the low temperatures $(150-300 \mathrm{~K})$ and the glass transition at $408 \mathrm{~K}$. The glassy storage modulus is on the order of $10^{9} \mathrm{~Pa}$ and the rubbery modulus is on the order of $10^{7} \mathrm{~Pa}$. The glass transition occurs over a temperature range of $10 \mathrm{~K}$. The model isochrone reproduces most of the important features of the experimental data. The large loss modulus peak height of the $\beta$ transition results from the choice of the functional form of the activation energy distribution function as well as the value of the bulk modulus $K$. Although a smaller value of $K$ would also produce a better match in the glassy storage modulus, we retain the same value due to the lack of more 
TABLE I. (a) Material parameters obtained from experimental measurements, (b) $f_{v}(T)$ function parameters, (c) $P\left(\Delta H_{c}\right)$ function parameters, and (d) parameters fitted from a selected isochrone.

\begin{tabular}{|c|c|c|}
\hline Parameter & Symbol & Value \\
\hline \multicolumn{3}{|l|}{ (a) } \\
\hline Bulk modulus & K & $3.3 \times 10^{9} \mathrm{~Pa}$ \\
\hline Strand length & $l_{0}$ & $1.85 \times 10^{-9} \mathrm{~m}$ \\
\hline Glassy Poisson ratio & $v_{\infty}$ & 0.4 \\
\hline Instantaneous Poisson ratio & $v_{0}$ & 0 \\
\hline \multicolumn{3}{|l|}{ (b) } \\
\hline Dilatometric glass transition temperature & $T_{g}$ & $387 \mathrm{~K}$ \\
\hline Thermal expansion coefficient below $T_{g}$ & $\alpha_{g}$ & $1.95 \times 10^{-4} \mathrm{~cm}^{3} /\left(\mathrm{cm}^{3}{ }^{\circ} \mathrm{C}\right)$ \\
\hline Thermal expansion coefficient above $T_{g}$ & $\alpha_{r}$ & $5.70 \times 10^{-4} \mathrm{~cm}^{3} /\left(\mathrm{cm}^{3}{ }^{\circ} \mathrm{C}\right)$ \\
\hline Free volume fraction at $0 \mathrm{~K}$ & $f_{v \mathrm{~h}}$ & 0 \\
\hline \multicolumn{3}{|l|}{ (c) } \\
\hline $\begin{array}{l}\text { Mean activation energy associated with the } \\
\beta \text { transition normal distribution }\end{array}$ & $\bar{H}_{c}$ & $70.7 \mathrm{~kJ} / \mathrm{mol}$ \\
\hline $\begin{array}{l}\text { Standard deviation of } \beta \text { relaxation normal } \\
\text { distribution }\end{array}$ & $\sigma_{\mathrm{Hc}}$ & $7.1 \mathrm{~kJ} / \mathrm{mol}$ \\
\hline $\begin{array}{l}\text { Decay constant of exponential decay } \\
\text { distribution }\end{array}$ & $H^{*}$ & $44 \mathrm{~kJ} / \mathrm{mol}$ \\
\hline \multicolumn{3}{|l|}{ (d) } \\
\hline \multicolumn{3}{|l|}{ Entropic force } \\
\hline $\begin{array}{l}\text { Number of strands per area } \\
\text { Frictional force }\end{array}$ & $n_{m}$ & $1.20 \times 10^{-4} \mathrm{moles} / \mathrm{m}^{2}$ \\
\hline Free volume fraction parameter, Eq. (17) & $b$ & 4.0 \\
\hline Characteristic friction coefficient, Eq. (17) & $\zeta_{0}$ & $1.0 \times 10^{-22} \mathrm{~kg} / \mathrm{s}$ \\
\hline \multicolumn{3}{|l|}{ Glassy relaxation } \\
\hline Rate constant prefactor, Eq. (26) & $B_{c 0}$ & $6 \times 10^{16} \mathrm{~s}^{-1}$ \\
\hline
\end{tabular}

reliable values. The large loss peak height of the $\alpha$ transition is higher than that of the experiment because the model accounts only for one relaxation process for the chain translational motion. This feature is inherent in the single subtube configuration chosen for the analysis. Multiple relaxation times resulting from inclusion of a distribution in the free volume fraction can decrease the peak height of the modulus.

The process of fitting the model predictions to a single isochrone of the material characterizes the resin completely for our purposes. The model can then be used to predict isochronal temperature sweeps at other frequencies as well as isothermal frequency sweeps at different temperatures [Simon and Ploehn (1997)]. In Sec. III, we examine the predictions of isochronal temperature sweeps for water-saturated samples of the same resin.

\section{Plasticized resin response}

Wang and Ploehn (1996) saturated the resin with water by immersion. The maximum water uptake in the resin was about $3.3 \%$ by weight of resin. This corresponds to about $4 \%$ by volume using the equation [Browning (1978)]

$$
V_{p}=\frac{1}{1+\left(\rho_{p} / \rho_{d}\right)(M / 100)},
$$

where $M$ is the weight percent of water in the resin and $\rho$ is the density of the component. We used a polymer specific gravity value of 1.28 [Browning (1978)] in Eq. (16). Using 

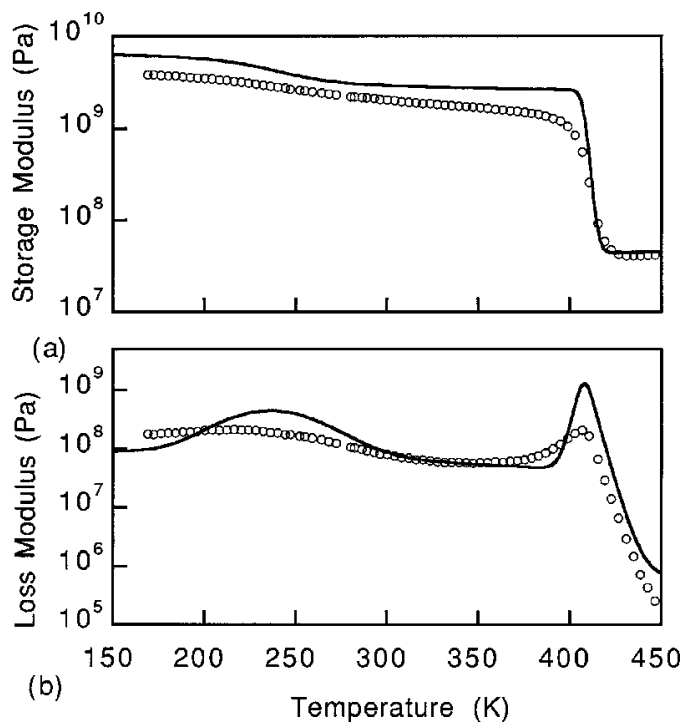

FIG. 1. Isochronal temperature sweep (10 rad/s) comparison between experimental dry resin data [circles, Wang and Ploehn (1996)] and the fitted predictions of the TJ model (solid curves), showing the storage moduli (a) and loss moduli (b).

Browning's (1978) hypothetical $T_{\mathrm{gd}}=4^{\circ} \mathrm{C}$ for water, we also assume that the free volume fraction contributed by water below $4{ }^{\circ} \mathrm{C}$ is zero. We anticipate that close to its normal freezing point, water provides very little mobility. We use the value of $\alpha_{d}=4$ $\times 10^{-4}{ }^{\circ} \mathrm{C}$ for water as successfully used by Browning (1978) for depression of the dilatometric $T_{g}$.

Figure 2 compares the predictions of the model for the plasticized resin with the experimental data for the WP resin. All the parameters in the model are those obtained previously by fitting the response to the dry resin data. The primary effect of the plasticizer through the friction coefficient is to decrease the glass transition temperature. We can see that the $\alpha$ transition temperature decreases by about $20 \mathrm{~K}$ just as in the experimental data. The dry resin prediction is also included in the plot for comparison. The plasticized material attains its rubbery zone at temperatures lower than that of the dry material. Nevertheless, the magnitude of the rubbery modulus remains essentially the same for both materials. We have not included the change in the rubbery modulus due to swelling of the polymer network. This effect would have to be included into the entropic force and will be most significant for gels. The experimental data for the epoxy resin shows very little swelling effect relative to the scatter in the data.

Figure 3 compares the predicted frequency responses for the plasticized material and the dry material. The curves are identical in shape, except that the plasticized resin manifests its full behavior over a frequency range shifted to higher values than that of the dry resin. We can see that the frequency of the glass transition increases with plasticization, while the frequency of the $\beta$ transition remains the same. This result, consistent with experimental observations, follows from our premise that the plasticizer only affects the friction coefficient associated with chain translational motion. The glassy relaxations are independent of the friction coefficient. 

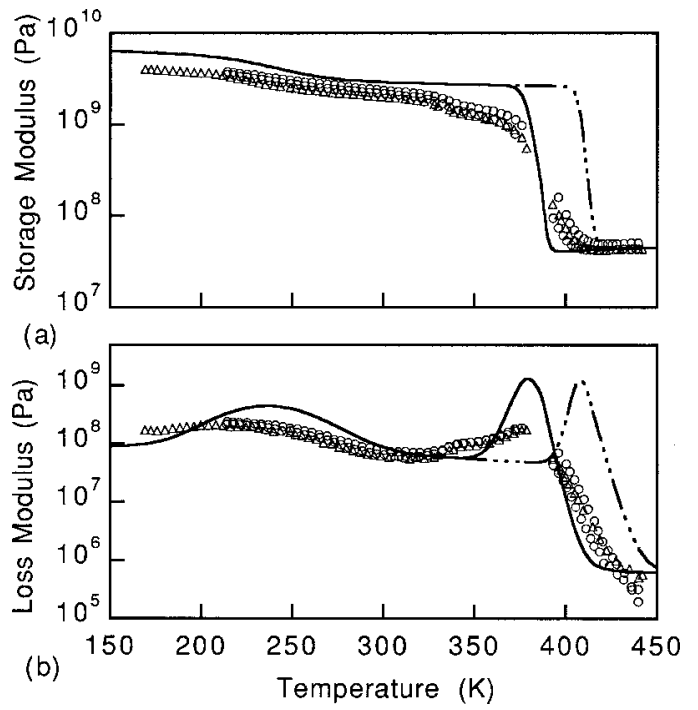

FIG. 2. Isochronal temperature sweep (10 rad/s) comparison between experimental wet resin data [various symbols representing duplicate experiments; Wang and Ploehn (1996)] and the predictions of the tube-junction model, showing the storage moduli (a) and loss moduli (b). Solid curves are predictions for a homogeneous plasticized resin, and dashed curves are predictions for the homogeneous dry resin shown in Fig. 1.

\section{Secondary effects on viscoelastic functions}

Closer inspection of the experimental data in Figs. 1 and 2 reveals a change in the shape of the temperature sweep profiles. Ferry (1980) notes similar changes for other polymers such as PVC plasticized with diethylhexyl succinate. These changes are duplicated in the theoretical curves. The loss modulus peak at the glass transition for the wet
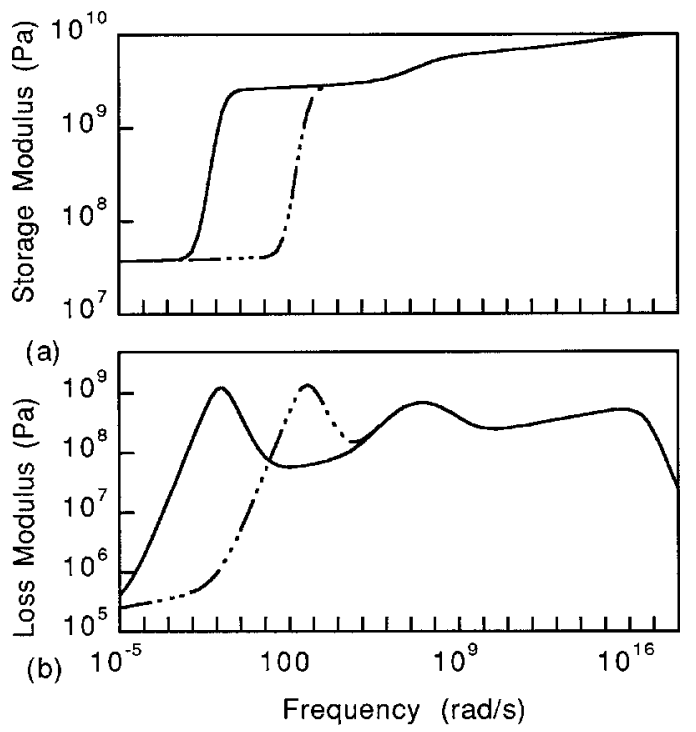

FIG. 3. Isothermal frequency sweep $(395 \mathrm{~K})$ predictions from the TJ model for the dry and wet resin characterized in Figs. 1 and 2, showing the storage moduli (a) and loss moduli (b). Solid curves are predictions for a homogeneous dry resin, and dashed curves are predictions for the homogeneous plasticized resin. 

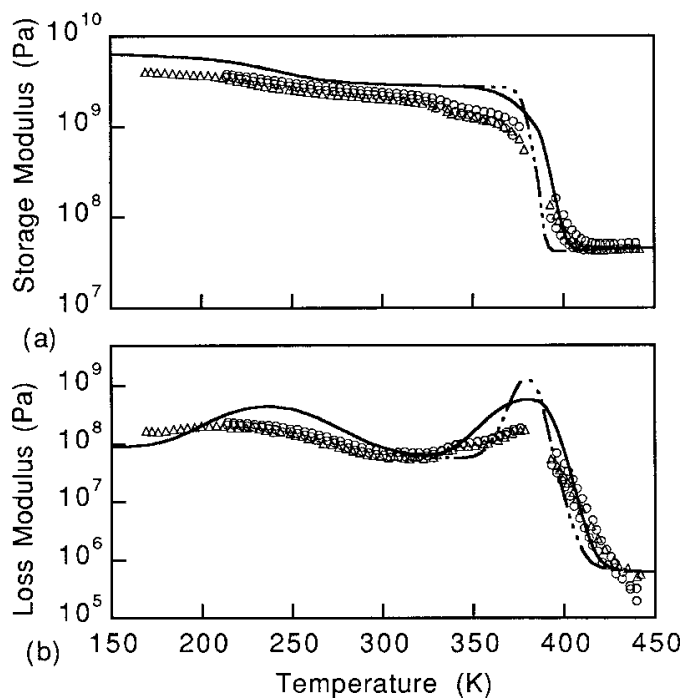

FIG. 4. Isochronal temperature sweep (10 rad/s) comparison between experimental wet resin data [various symbols representing duplicate experiments, Wang and Ploehn (1996)] and the predictions of the tube-junction model, showing the storage moduli (a) and loss moduli (b). Solid curves are predictions for an inhomogeneous resin, and dashed curves are predictions for a homogeneous resin.

resin decreases in height and increases in breadth compared to that for dry resin. The slope of the storage modulus curve through the glass transition decreases slightly relative to the dry resin, consistent with the broadening on the loss modulus peak.

Qualitatively better predictions can be achieved if we assume that the plasticized resin contains a distribution (or a relative increase in the distribution) of the free volume fraction. In Fig. 4 we plot the previous data with the prediction of the model for an inhomogeneous resin. In this calculation, we assume that Eq. (14) gives the mean free volume fraction that is used in the gamma distribution in Eqs. (11) and (12).

Robertson (1992) calculates fractional free volumes and the mean squared fluctuations in free volumes using the Simha-Somcynsky equations. Calculations for polystyrene show values of mean square fluctuations that are about $0.4 \%$ of the absolute values of the fractional free volumes. This corresponds to a standard deviation that is about $20 \%$ of the fractional free volume. For our system, we are interested in the standard deviation of free volume fraction associated with water in the epoxy resin. Although this value may be different from the standard deviation of the total free volume fraction as calculated by Robertson, we assume that a value within this range is acceptable for illustrative purposes. A standard deviation that is $5 \%$ of the fractional free volume $(0.005$ out of approximately 0.1 ) gives a response that is fairly close to the experimental data (Fig. 4). Other values may provide a better " fit,", but we do not attempt to regress this value from the data using our model. This should be attempted only if we had knowledge of measured distribution functions of water within the epoxy resin.

The modulus is a volume-averaged quantity obtained using Eq. (9). The trend in the shape change in the model is similar to that in the experimental data. In comparison to the original no-distribution curve, the steepness of the storage modulus curve with distribution is smaller, giving more rounded profile than for the dry. This qualitative trend is obvious in the experimental data of Wang and Ploehn as well as in the data shown by Ferry (Figure 17-44, 1980). 


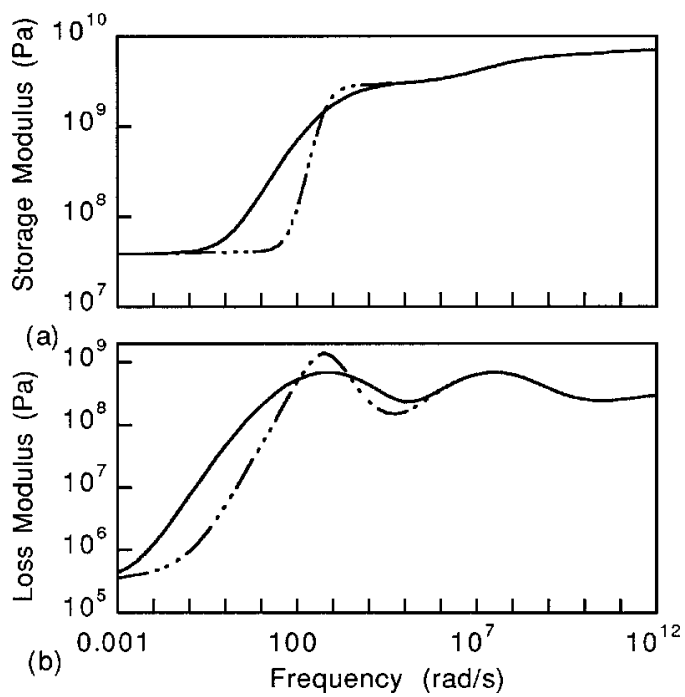

FIG. 5. Isothermal frequency sweep $(395 \mathrm{~K})$ predictions from the TJ model for the homogeneous and inhomogeneous wet resins characterized in Fig. 4, showing the storage moduli (a) and loss moduli (b). Solid curves are predictions for an inhomogeneous resin, and dashed curves are predictions for a homogeneous resin.

With respect to the change in shape of the frequency sweep response, Ferry notes (p. $535,1980)$ that the storage modulus changes more gradually with frequency and the maximum in the loss modulus is less sharp. These trends are also obtained from the model for the frequency response in Fig. 5, where we plot the frequency sweeps with and without the free volume distributions. These curves correspond to the same parameters used for the curves in Fig. 4, at a reference temperature of $395 \mathrm{~K}$. The exact shape of the profiles will depend on the assumed shape of the distribution function and the magnitude of the standard deviation.

Figures 4 and 5 indicate that all of the secondary effects observed in plasticization, namely the spreading of the glass transition over a larger temperature and frequency range, may be related directly to an increase in the spread of the free volume distribution in the material. A distribution in the tube orientations in the strain field, which we neglected in the model, can only produce spreading in the frequency sweep, but not in the temperature sweep. This observation may suggest that the plasticization of the resin is not completely homogeneous in space. In other words, the model predictions better represent the experimental data when the free volume in the model has a nonhomogeneous spatial distribution. The fluctuation in the values of free volume at different locations probably stems from fluctuations in the concentrations of the plasticizer (water) or from differences in the interactions of the plasticizer with different sections of the polymer chain. At this stage, we cannot speculate further on the exact source of the inhomogeneity because the model does not incorporate a more detailed description. The existence of a free volume distribution in pure materials has been detected experimentally [Victor and Torkelson (1987, 1988); Cicerone et al. (1995)]. Our calculations suggest that the presence of plasticizer amplifies the effect of free volume heterogeneities on dynamic mechanical properties.

A plot of the relaxation times in Fig. 6, calculated using an approximate difference formula [Ferry (1980)], shows the shift in the distribution of relaxation times with plasticization at $395 \mathrm{~K}$. At low time scales, the relaxation time distribution associated with the $\beta$ transition goes through a maximum. The shape of the profile is similar to the shape 


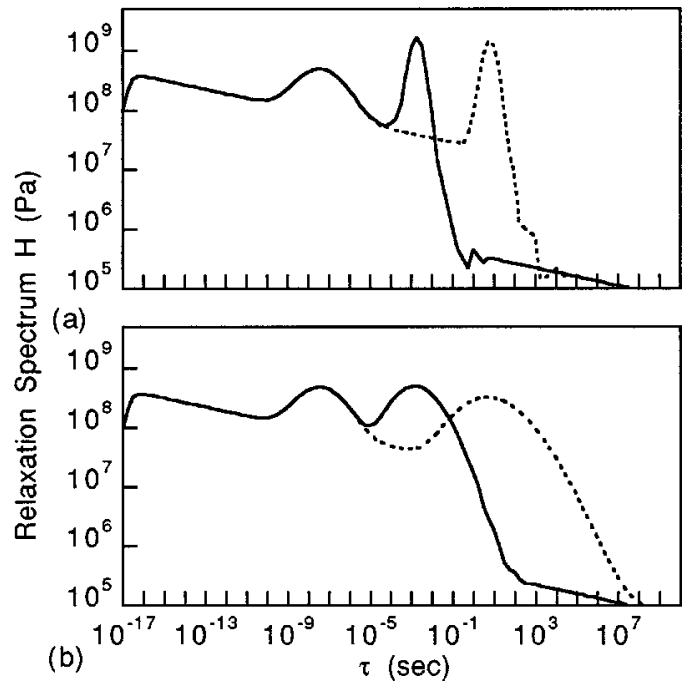

FIG. 6. Relaxation spectra $H$ of the TJ model at $395 \mathrm{~K}$ for dry epoxy resin and $4 \%$ water saturated resin, assuming (a) homogeneous and (b) inhomogeneous free volume distributions. Solid and dashed curves are predictions for plasticized and dry resins, respectively.

of the activation energy distribution. The glass transition peak, associated with reptative motion of the chain, broadens and decreases in height with the introduction of the free volume distribution. The decrease in the relaxation times due to the additional free volume of the plasticizer is as expected.

\section{CONCLUSIONS}

The tube-function model provides a tool to examine the effect of plasticizers on the viscoelastic behavior of crosslinked polymers such as epoxy resins. We used the additivity of the free volume fractions, as used in the Kelley-Bueche theory, to model the increase in free volume fraction with increase in plasticizer content. This approximation produced a prediction of water-mediated shift of the viscoelastic glass transition temperature to within experimental error of the measurements with no adjustments to the model parameters.

We have shown that the decrease in the viscoelastic glass transition temperature could be attributed directly to the decrease in the friction coefficient through the free volume fraction. Although these ideas have always been accepted in the context of the WLF equation and the Kelley-Bueche model, an explicit prediction of the change in the viscoelastic profile of the material as a function of temperature and plasticizer has not been published previously. The model, furthermore, predicts an increase in the glass transition frequency in isothermal frequency sweeps in agreement with observed polymer behavior.

We have shown that the typical changes in the shape of the modulus profiles of plasticized epoxy resin can be explained by an increase in the distribution of the free volume fraction. These results suggest that plasticization may not be uniform on the microscopic level, at least for low values of absorption. Water may be distributed inhomogeneously within the polymer, or it may interact differently with various parts of the polymer chain to provide a distribution in the frictional coefficients. Both factors can lead to a distribution of free volume fraction. 


\section{ACKNOWLEDGMENTS}

The authors wish to thank the U.S. National Science Foundation for financial support provided through Grant No. CTS-9258137 and the Offshore Technology Research Center (Grant No. CDR-8721512), and the U.S. Department of Energy for support provided through Grant No. DE-FC02-91ER75666.

\section{References}

Adam, G. and J. H. Gibbs, "On the temperature dependence of cooperative relaxation properties in glassforming liquids," J. Chem. Phys. 43, 139-146 (1965).

Astarita, G., M. E. Paulaitis, and R. G. Wissinger, "Thermodynamics of the glass transition," J. Polym. Sci., Part B: Polym. Phys. 27, 2105-2116 (1989).

Browning, C. E., "The mechanisms of elevated temperature property losses in high performance structural epoxy resin matrix materials after exposures to high humidity environments,' Polym. Eng. Sci. 18, 16-24 (1978).

Browning, C. E., G. E. Husman, and J. M. Whitney, "Moisture effects in epoxy matrix composites," in Composite Materials: Testing and Design (Fourth Conference), ASTM STP 617 (American Society for Testing and Materials, Philadelphia, PA, 1977), pp. 481-496.

Cicerone, M. T., F. R. Blackburn, and M. D. Ediger, "Anomalous diffusion of probe molecules in polystyrene: evidence for spatially heterogeneous segmental dynamics," Macromolecules 28, 8224-8232 (1995).

Cohen, M. L. and G. S. Grest, 'Liquid-glass transition, a free-volume approach,” Phys. Rev. B 20, 1077-1098 (1979).

Cohen, M. L. and G. S. Grest, “A new free-volume theory of the glass transition,” Ann. (N.Y.) Acad. Sci. 371, 199-209 (1981).

Cohen, M. L. and G. S. Grest, "The nature of the glass transition," J. Non-Cryst. Solids 61 \& 62 , 749-760 (1984).

DiMarzio, E. A., "Equilibrium theory of glasses," Ann. (N.Y.) Acad. Sci. 371, 1-20 (1981).

Donth, E. J., Relaxation and Thermodynamics of Polymers-Glass Transition (Akademie Verlag GmbH, Berlin, 1992).

Ferry, J. D., Viscoelastic Properties of Polymers (Wiley, New York, 1980).

Gibbs, J. H. and E. A. DiMarzio, "Nature of the glass transition and the glassy state," J. Chem. Phys. 28 $373-383$ (1958).

Gupta, V. B., L. T. Drzal, C. Y-C. Lee, and M. J. Rich, "The effects of stoichiometry and structure on the dynamic torsional properties of a cured epoxy resin system,"' J. Macromol. Sci., Phys. 23, 435-466 (1985).

Kauzmann, W., "The nature of the glassy state and the behavior of liquids at low temperatures," Chem. Rev. 43, 219-256 (1948).

Kelley, F. N. and F. J. Bueche, "Viscosity and Glass Temperature Relations for Polymer-Diluent Systems," J. Polym. Sci. 50, 549-556 (1961).

Moynihan, C. T. and A. V. Lesikar, "Comparison and Analysis of Relaxation Processes at the Glass Transition Temperature," Ann. (N.Y.) Acad. Sci. 371, 151-159 (1981).

Roberts, G. E. and E. F. T. White, "Relaxation processes in amorphous polymers," in The Physics of Glassy Polymers, edited by R. N. Haward (Wiley, New York, 1973).

Robertson, R. E., "Free-volume theory and its application to polymer relaxation in the Glassy State," Computational Modeling of Polymers, edited by J. Bicerano (Marcel Dekker, Inc., New York, 1992).

Simha, R., "Configurational thermodynamics of the liquid and glassy polymeric states," Macromolecules 10, 1025-1030 (1977).

Simha, R. and T. Somcynsky, "On the statistical thermodynamics of spherical and chain molecule fluids,' Macromolecules 2, 342-350 (1969).

Simon, P. P. and H. J. Ploehn, "Molecular-level modeling of the viscoelasticity of crosslinked polymers-Effect of time and temperature," J. Rheol. 41, 641-670 (1997).

Victor, J. G. and J. M. Torkelson, "'On measuring the distribution of local free volume in glassy polymers by photochromic and fluorescence techniques," Macromolecules 20, 2241-2250 (1987).

Victor, J. G. and J. M. Torkelson, "Photochromic and fluorescent probe studies in glassy polymer matrices. 3. Effects of physical aging and molar weight on the size distribution of local free volume in polystyrene,' Macromolecules 21, 3490-3497 (1988).

Wang, J-Y. and H. J. Ploehn, "Dynamic mechanical analysis of the effect of water on glass bead-epoxy composites," J. Appl. Polym. Sci. 59, 345-357 (1996).

Williams, M. L., R. F. Landel, and J. D. Ferry, "The temperature dependence of relaxation mechanisms in amorphous polymers and other glass-forming liquids," J. Am. Chem. Soc. 77, 3701-3707 (1955). 